\title{
Grain Size and Pressure Effects on Spall Strength in Copper
}

A. J. Schwartz, J. U. Cazamias, P. S. Fiske, R. W. Minich

This article was submitted to

$12^{\text {th }}$ Biennial International Conference on the APS Topical Group on Shock Compression on Condensed Matter, Atlanta, GA, June 2429, 2001

\section{June 1, 2001}

Lawrence

Livermore

National

Laboratory 


\section{DISCLAIMER}

This document was prepared as an account of work sponsored by an agency of the United States Government. Neither the United States Government nor the University of California nor any of their employees, makes any warranty, express or implied, or assumes any legal liability or responsibility for the accuracy, completeness, or usefulness of any information, apparatus, product, or process disclosed, or represents that its use would not infringe privately owned rights. Reference herein to any specific commercial product, process, or service by trade name, trademark, manufacturer, or otherwise, does not necessarily constitute or imply its endorsement, recommendation, or favoring by the United States Government or the University of California. The views and opinions of authors expressed herein do not necessarily state or reflect those of the United States Government or the University of California, and shall not be used for advertising or product endorsement purposes.

This is a preprint of a paper intended for publication in a journal or proceedings. Since changes may be made before publication, this preprint is made available with the understanding that it will not be cited or reproduced without the permission of the author.

This work was performed under the auspices of the United States Department of Energy by the University of California, Lawrence Livermore National Laboratory under contract No. W-7405-Eng-48.

This report has been reproduced directly from the best available copy.

Available electronically at http://www.doc.gov/bridge

Available for a processing fee to U.S. Department of Energy

And its contractors in paper from

U.S. Department of Energy

Office of Scientific and Technical Information

P.O. Box 62

Oak Ridge, TN 37831-0062

Telephone: (865) 576-8401

Facsimile: (865) 576-5728

E-mail: reports@adonis.osti.gov

Available for the sale to the public from

U.S. Department of Commerce

National Technical Information Service

5285 Port Royal Road

Springfield, VA 22161

Telephone: (800) 553-6847

Facsimile: (703) 605-6900

E-mail: orders@ntis.fedworld.gov

Online ordering: http://www.ntis.gov/ordering.htm

OR

Lawrence Livermore National Laboratory

Technical Information Department's Digital Library

http://www.llnl.gov/tid/Library.html 


\title{
GRAIN SIZE AND PRESSURE EFFECTS ON SPALL STRENGTH IN COPPER
}

\author{
Adam J. Schwartz, James U. Cazamias, Peter S. Fiske, and Roger W. Minich \\ Lawrence Livermore National Laboratory \\ L-355, 7000 East Avenue \\ Livermore, $C A 94550$
}

\begin{abstract}
We are executing a systematic study to quantify the effects of specific microstructural features on the spall behavior of $99.999 \%$ copper. Single crystals with [100] orientation, polycrystals with three grain sizes, and internally oxidized single crystals are shocked with $\mathrm{Cu}$ flyers at velocities from 300 to 2000 $\mathrm{m} / \mathrm{s}$ using a $35-\mathrm{mm}$ single/two-stage light gas gun. VISAR measurements of the free surface velocity are used to characterize the spall pullback signal and details of the ringing. The high purity single crystals exhibit the highest spall strength followed by the large, medium and small grain size polycrystalline samples. $\mathrm{Cu}-0.15 \mathrm{wt} . \% \mathrm{Si}$ single crystals have been internally oxidized to produce a fine dispersion of $350 \mathrm{~nm}$ silica particles. These samples exhibit the lowest spall strength, a factor of two and greater below the high purity single crystals.
\end{abstract}

\section{INTRODUCTION}

The spall process is a series of complex phenomena that includes nucleation of voids, growth of voids, coalescence/interaction of voids and separation/spall. When a compressive shock is introduced into a material via a high explosive or plate impact, the interaction of rarefaction waves can generate a tensile pulse. If the magnitude of this tensile pulse locally exceeds a critical threshold stress, voids or cracks can nucleate. When such void or crack networks link together, spall failure results. If voids nucleate and grow, but do not coalesce leading to full separation, this is termed incipient spall.

It is now widely accepted that the microstructure of a material can exert a strong influence on spall strength. For example, experiments on high-purity copper reported by Kanel, Rasorenov and Fortov (1) indicate the spall strength of single-crystal copper is a factor of two to three times higher than commercial purity polycrystalline copper. Although details of the purity of the single and polycrystalline materials are not provided, these results clearly indicate the potent effects that the presence of grain boundaries or impurities exert on the nucleation, growth, and coalescence of voids in materials. Results of recovered electron-beam spalled polycrystalline $\mathrm{Cu}$ from Lassila (2) further illustrate this effect. Void nucleation is observed along a relatively narrow plane with coalescence along certain grain boundaries and triple junctions. It is important to recognize that preferential void nucleation is not observed on specific grain boundaries.

Seaman, et al. $(3,4)$ have executed one of the most comprehensive spall studies to date. This work in the late 1960's and early 1970's resulted in spall models for ductile and brittle failures. One of their most significant results concerns the work on various grades of aluminum, including high purity, $1145 \mathrm{Al}$ and 2024-T81 Al. They found that the threshold stress for void nucleation is not strictly a function of the yield strength; the high purity $\mathrm{Al}$, which has much lower yield strength, exhibited a damage threshold higher than that of the $1145 \mathrm{Al}$. The 2024-T81 alloy, solution treated and aged to improve strength and corrosion properties exhibits significantly different damage behavior. Fractures nucleate by cracking of the inclusions, and 
propagate along planes of high inclusion density. These significant experiments on various grades of Al show that failure is a strong function of both the precipitate distribution as well as the intrinsic properties of the alloy. An investigation by Butcher (5) has determined the incipient spall thresholds for 6061-T6 Al annealed to produce $\mathrm{Cu}$ platelet precipitates and found that the orientation of the high density precipitate planes dictated the critical pressure for spall failure. These works conclude that the inclusion or precipitate distribution largely controls the spall strength and that if the inclusions are removed, higher spall strengths would be observed.

Other works have examined the influence of grain size on spall strength. In these cases, conflicting experimental results occur. In some cases, spall strength is observed to increase with decreasing grain size, analogous to the Hall-Petch relation, which correlates yield strength with grain size. Other experiments indicate higher spall strengths with increasing grain size.

We are undertaking a coupled experimental/modeling investigation to elucidate the effects of specific microstructural features on the spall behavior. Our objectives are to provide quantitative relationships between specific microstructural features (grain boundaries, inclusions, etc.) and effects on spall behavior, develop and experimentally validate spall criteria and models in which the effects of microstructure are included, and elucidate fundamental mechanisms of void nucleation, growth, and coalescence for specific microstructural details. In this paper, we report the results on single crystals, high purity polycrystals, and preliminary data on internally oxidized samples.

\section{APPROACH}

The approach is to develop engineered microstructures that isolate effects of specific microstructural features (grain size, inclusion size and distribution, etc.). Material systems are chosen based on the ability to develop particular size and shape distributions for the feature of interest. It is anticipated that five specific test microstructures will be examined; (1) high-purity single crystal without inclusions, (2) high-purity single crystal with inclusions, (3) high-purity polycrystal without inclusions, (4) high-purity polycrystal with inclusions, and (5) material with grain boundary phase. Quantitative microstructural characterization is performed by optical, scanning electron microscopy (SEM), and transmission electron microscopy (TEM) if necessary to determine the volume fractions of inclusions, grain size and distribution, etc. Quantification of the microstructure is crucial in developing the microstructurally based models. For example, a range of inclusion volume fractions and size distributions will be engineered while keeping other microstructural features constant, and tested in the gas gun under identical conditions. With this approach, the quantitative correlations between specific microstructural features and the effects on spall behavior will be obtained. The evolving void size distributions required by theory will be obtained by selective recovery and quantitative microscopy. Gas gun experiments are conducted over a range of impact velocities to control the pressure of the shock. Wave profiles, determined by VISAR are used to capture the free surface velocity-time history and will provide details of the failure mechanism.

\section{MATERIALS}

99.999\% Cu cold-rolled polycrystalline plate material was procured from Plasmaterials (6) with dimensions $304 \mathrm{~mm}$ x $152 \mathrm{~mm}$ x $9.5 \mathrm{~mm}$. The plate was subjected to an additional cold rolling procedure to reduce the thickness to $6.4 \mathrm{~mm}$ followed by a recrystallization study to determine appropriate conditions to produce a wide range of grain sizes. To produce a suite of specimens with various grain sizes, samples were annealed in vacuum at $300^{\circ} \mathrm{C}$ for one hour to produce an average grain size of $8.6 \mu \mathrm{m}, 650^{\circ} \mathrm{C}$ for one hour to produce a grain size of $50 \mu \mathrm{m}$, and at $800^{\circ} \mathrm{C}$ for 24 hours to produce an average grain size of $133 \mu \mathrm{m}$. Optical light micrographs of these samples are shown in Fig. 1.

Single crystals of $99.999 \% \mathrm{Cu}$ and $\mathrm{Cu}+0.15$ wt.\% Si were obtained from Metal Crystals and Oxides (7) in the form of $25 \mathrm{~mm}$ diameter by 100 $\mathrm{mm}$ long. The silicon-containing specimens are 
sectioned to $5 \mathrm{~mm}$ thickness discs and exposed to a contained atmosphere of $\mathrm{Cu}$ powder and $\mathrm{Cu}_{2} \mathrm{O}$ at $1300 \mathrm{~K}$ for 24 hours. The process produces a fine dispersion of $\mathrm{SiO}_{2}$ particles with size $\sim 350 \mathrm{~nm}$.

Gas gun experiments are performed on a $35 \mathrm{~mm}$ single/two-stage light gas gun with $\mathrm{Cu}$ flyer plates at 300 to $2000 \mathrm{~m} / \mathrm{s}$. Velocity-time histories are acquired with VISAR.
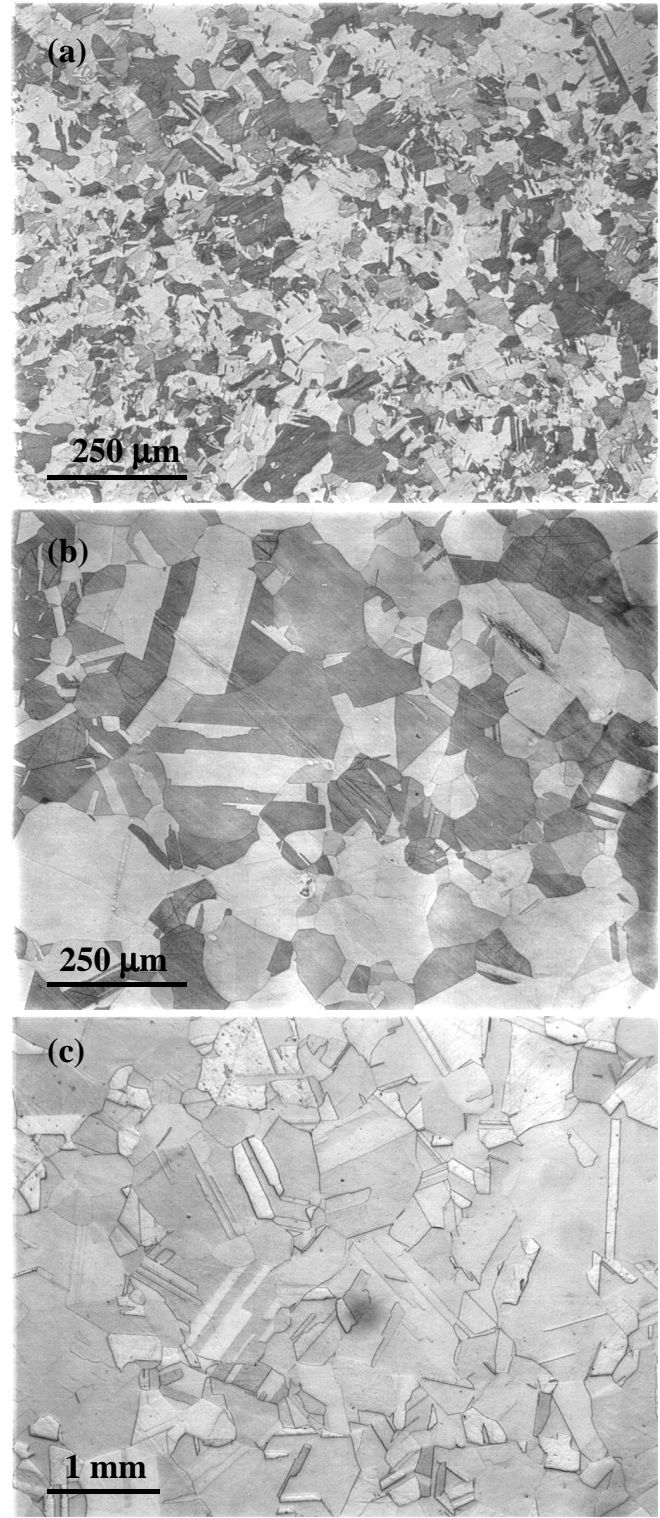

FIGURE 1. Optical light micrographs of the $99.999 \% \mathrm{Cu}$ processed to modify the grain size, (a) $8.6 \mu \mathrm{m}$, (b) $50 \mu \mathrm{m}$, and (c) $133 \mu \mathrm{m}$.

\section{RESULTS AND DISCUSSION}

Figure 2 shows the velocity-time profiles for the [100] single crystals and $50 \mu \mathrm{m}$ grain size polycrystals. For all samples, the frequency of the reverberations increases with impact stress; however, above free surface velocities of $1 \mathrm{~km} / \mathrm{s}$, the increase cannot be attributed to increasing sound speed, and we attribute the frequency to multiple spall planes. The single crystal data exhibit a spike following the initial pullback, which to our knowledge, has not been observed previously.
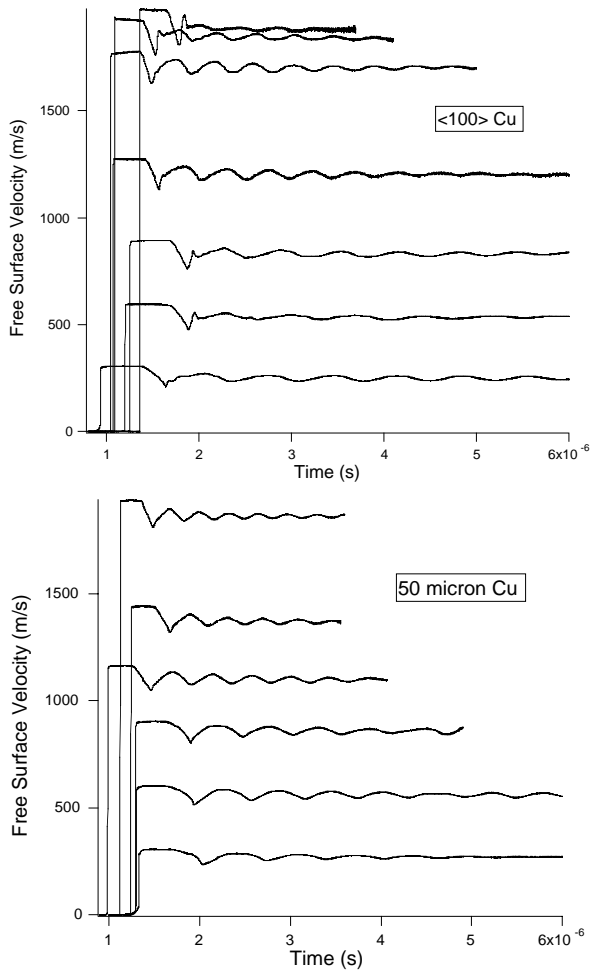

FIGURE 2. Pullback velocity versus time for the [100] single crystal and polycrystal.

The pullback velocity (spall strength) as a function of pressure has been measured for the [100] single crystals, polycrystals, and two internally oxidized samples. The results are shown in Fig. 3. The [100] single crystal exhibits increasing spall strength with increasing impact pressure as well as the highest spall strength of all the specimens. However, a comparison of the pullback velocity for these samples to those reported by Kanel (1) indicate a much lower spall 
strength in this case. Back Laue $\mathrm{x}$-ray diffraction indicates the presence of a number of low angle grain boundaries giving rise to a spread in orientation of approximately 2 degrees. Kanel did not report either the purity or spread in orientation.

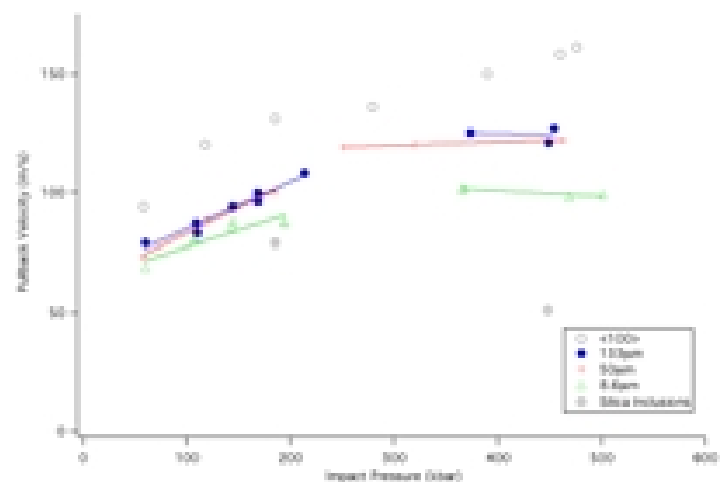

FIGURE 3. Pullback velocity versus impact pressure for the [100] single crystal and polycrystal.

Data from the $99.999 \% \mathrm{Cu}$ polycrystals indicate an increase spall strength up to a certain threshold, followed by plateau during which the spall strength remains constant with increasing pressure. This behavior is consistent with the results of Buchar, et al. (8) and Jacobi, et al. (9). Jacobi et al.'s data however, reaches a plateau at values below our lowest pressure. Our data indicates that the large grain size material exhibits higher spall strength than the small grain size material. This is in opposition to the work by Buchar, et al. (8), and Christy, et al. (10). Buchar used $99.96 \% \mathrm{Cu}$, Christy tested $99.99 \% \mathrm{Cu}$. This difference in purity may be partially responsible for the inverse relationship of grain size and spall strength. Jacobi, et al. (9) report decreasing spall strength with increasing impurity content. $\mathrm{The} \mathrm{Cu}$ 0.15 wt.\% Si [100] single crystals exhibit the lowest pullback signal. This indicates the potent nucleation potential of the small, hard silica particles. There is also a rate effect buried in our data. The higher stress data undergo larger strain rates under loading and release, so the increase in the pullback velocity may also be attributed to strain rate.

\section{SUMMARY}

Engineered microstructure are used to quantify the influence on spall behavior. Systematic gas gun experiments and microstructural characterzation on [100] single crystals, $99.999 \% \mathrm{Cu}$ polycrystals, and internally oxidized $\mathrm{Cu}-\mathrm{Si}$ single crystals. The single crystals have the highest spall strength followed by the large, medium, and small polycrystals. The single crystals containing a fine dispersion of silica particles exhibit the lowest spall strength.

\section{ACKNLOWEDGMENTS}

This work was performed under the auspices of the U.S. Department of Energy by the University of California, Lawrence Livermore National Laboratory under contract No. W-7405-Eng-48.

\section{REFERENCES}

1. Kanel, G.I., Rasorenov, S.V., and Fortov, V.E., "The Dynamic Strength of Copper Single Crystals," in ShockWave and High-Strain-Rate Phenomena in Materials, edited by M.A. Meyers et al., Marcel Dekker, New York, 1992, pp. 775-782.

2. Lassila, D.H., unpublished research

3. Seaman, L., Barbee, Jr., T.W., and Curren, D.R., "Dynamic Fracture Criteria of Homogeneous Materials," Air Force Weapons Laboratory Technical Report No. AFWL-TR-71-156, 1972.

4. Curran, D.R.,. Seaman, L., and Shockley, D.A., Physics Reports, 147, No. 5 and 6, 253-388, (1987).

5. Butcher, B.M., Spallation of 6061-T6 aluminum: Behavior of Dense Media Under High Dynamic Pressure, Gordon and Breach, New York, 1968.

6. Plasmaterials, Inc. Pleasanton, CA, USA.

7. Metal Crystals \& Oxides LTD, Cambridge, England.

8. Buchar, J., Elices, M., and Cortez, R., J. de Physique IV, C3, 1 C3-623-C3-630 (1991).

9. Jacobi, S., Zaretsky, E., and Shvarts, D., "Experimental study and modeling of dynamic fracture of copper," in Fundamental Issues and Applications of Shock-Wave and High-Strain-Rate Phenomena, edited by K.P. Staudhammer, et al., Elsevier Science, Oxford, 2001, pp. 525-531.

10. Christy, S., Pak, H.-R., and Meyers, M.A., "Effect of Metallurgical Parameters on Dynamic Fracture by Spalling of Copper," in Metallurgical Applications of Shock-Wave and High-Strain-Rate Phenomena, edited by L.E. Murr et al., Marcel Dekker, New York, 1986, pp. 835-863. 